\title{
MicroRNA-187 is an independent prognostic factor in lung cancer and promotes lung cancer cell invasion via targeting of PTRF
}

\author{
JIE PENG $^{1 *}$, HONG-ZHOU LIU ${ }^{2 *}$, JUN ZHONG ${ }^{3 *}$, ZHUO-FENG DENG $^{1}$, CHAO-RONG TIE ${ }^{4}$, QIAN RAO ${ }^{1}$, \\ WEI XU ${ }^{1}$, TAO YOU ${ }^{1}$, JING LI $^{1}$, CHUAN-BAO CAI ${ }^{1}$, QIAN LU ${ }^{1}$, WEI LIU ${ }^{1}$, YAN ZHANG ${ }^{1}$ and ZHI-YUN LEI ${ }^{1}$ \\ ${ }^{1}$ Department of Stomatology, Wuhan Medical and Healthcare Center for Women and Children, Wuhan, \\ Hubei $430015 ;{ }^{2}$ Center for Gene Diagnosis, and Departments of ${ }^{3}$ Second Oncology Surgery and \\ ${ }^{4}$ Stomatology, Zhongnan Hospital of Wuhan University, Wuhan, Hubei 430071, P.R. China
}

Received March 14, 2016; Accepted April 15, 2016

DOI: $10.3892 / o r .2016 .5083$

\begin{abstract}
MicroRNAs (miRNAs) are involved in the progression of different types of cancers giving new hope for cancer treatment. The role and regulatory mechanism of microRNA-187 (miR-187) are largely unknown. In the present study, 74 patients with non-small cell lung cancer (NSCLC) were selected. Tumor tissues and matched normal tissues were collected for determining the expression level of miR-187. Cell research was performed to detect the function of miR-187. The expression level was measured and miR-187 was found to be overexpressed in the NSCLC cell lines and tissues. Overexpression of miR-187 promoted cell proliferation in the A549 and H1650 cell lines. Moreover, overexpression of miR-187 also promoted cell migration and invasion. Polymerase I and transcript release factor (PTRF) was identified as a target of miR-187. Overexpression of miR-187 suppressed the expression of PTRF. Knockdown of PTRF promoted lung cancer cell invasion, and overexpression of PTRF had a negative effect on lung cancer cell invasion. The PTRF messenger RNA (mRNA) levels in cancer tissues were significantly lower than those in their adjacent normal lung tissues as determined by real-time PCR (RT-PCR). The expression of the PTRF protein was significantly weaker than that in the adjacent normal lung tissues using immunohistochemical staining. The findings revealed that miR-187 promotes cell growth and invasion by targeting PTRF and miR-187 may be a new prognostic factor for NSCLC.
\end{abstract}

Correspondence to: Dr Zhi-Yun Lei, Department of Stomatology, Wuhan Medical and Healthcare Center for Women and Children, 100 Hongkong Road, Wuhan, Hubei 430015, P.R. China E-mail:pjlhz@sina.com

*Contributed equally

Key words: non-small cell lung cancer, microRNA, microRNA-187, PTRF, prognostic factor

\section{Introduction}

Lung cancer is one of the most life-threatening tumors and is one of the leading causes of cancer-related deaths in China (1-3). Newly diagnosed lung cancer cases are mostly non-small cell lung cancers (NSCLCs) and the 5-year post-surgery survival rate is poor $(1,4,5)$. In the past few decades, strategies for the treatment of NSCLC, such as surgical resection, have improved. However, the prognosis and survival rate have not been effectively improved (6-8). The discovery of biomarkers, such as microRNAs (miRNAs), can be used for early screening and diagnosis.

miRNAs are non-coding small RNAs, $\sim 22$ nucleotides in length. miRNAs can regulate gene expression by binding to the seed sites of messenger RNA (mRNA) targets (4-7). Some studies suggest that miRNAs play important roles in a variety of biological processes, such as migration and invasion (3-6). Many reports suggest that miRNAs are dysregulated in various types of cancers such as gastric cancer, renal cell carcinoma, and lung cancer (8-10). Some studies have indicated that miR-24, miR-31, miR-133, and miR-342 are dysregulated in NSCLC (11-13).

Previous studies have shown that microRNA-187 (miR-187) plays an important role in cell invasion, proliferation, and migration in breast and lung cancer, leukemia, and ovarian cancer (14-18). The results are conflicting among the reported studies. In this study, the expression and function of miR-187 were investigated in NSCLC patient tissues and cell lines. The expression of miR-187 was increased in human NSCLC cell lines and tissues, and the overexpression of miR-187 enhanced NSCLC cell proliferation and invasion in the cell lines. Polymerase I and transcript release factor (PTRF) as a direct target of miR-187 was identified.

\section{Materials and methods}

Ethical approval. The research was approved by the Ethics Committee of Zhongnan Hospital of Wuhan University and complied with the Declaration of Helsinki. Written informed consent was obtained from all participants. The methods were carried out in accordance with the approved guidelines and regulations. 
Clinical tissues and cell lines. Clinical cancer and normal tissues were collected at the time of surgery. The patients had not received any treatment before surgery. All tissues were placed in liquid nitrogen until use. All NSCLC cell lines (H1650 and A549) and normal human bronchial epithelial cells (BEAS-2B) were obtained from the American Type Culture Collection (ATCC; Manassas, VA, USA). Cells were cultured in RPMI-1640 medium supplemented with $10 \%$ fetal bovine serum (FBS) and penicillin and streptomycin were added to the cell culture following ATCC-recommended conditions.

Plasmids, small interfering RNAs (siRNAs), and transfection. The miR-187 mimic was: forward, 5'-UCGUGUCUUGUG UUGCAGCCGG and reverse, 5'-GGCUGCAACACAAGA CACGAUU. The miR-187 mimic negative control was: forward, 5'-UUCUCCGAACGUGUCACGU and reverse, 5'-ACGUGACACGUUCGGAGAA. The miR-187 inhibitor (5'-CCGGCUGCAACACAAGACACGA) and miR-187-inhibitor negative control (5'-UUCUCCGAACGUGUCACGU) were designed and synthesized by Invitrogen Life Technologies (Shanghai, China). The siRNA of PTRF (PTRF-siRNA) and its negative control were chemically synthesized by Guangzhou RiboBio Co., Ltd. (Guangzhou, China). The mRNA of PTRF was cloned from complementary DNA (cDNA) into a pCMV vector using PCR (from our laboratory). Plasmids of PTRF, miR-411 mimic, miR-411 inhibitor, PTRF-siRNA, and their negative control were transfected using Lipofectamine 2000 Reagent (Life Technologies) according to the manufacturer's instructions.

Quantitative real-time PCR (RT-PCR). Total RNA was extracted with TRIzol reagent (Invitrogen, Carlsbad, CA, USA) and cDNA was synthesized using the RevertAid First Strand cDNA Synthesis kit (Fermentas, Burlington, ON, Canada). The expression levels of the PTRF gene were detected using RT-PCR on a CFX96 Real-Time PCR Detection system (Bio-Rad) using SYBR Green PCR master mix (Applied Biosystems). The samples were assayed in triplicate and glyceraldehyde-3-phosphate dehydrogenase (GAPDH) was used as an internal control to normalize the results. Relative gene expression levels were determined using the $2^{-\Delta \Delta \mathrm{Ct}}$ method. The PTRF primers were: forward, 5'-AATACGGTGAGCAAGCTGCTGGAG-3' and reverse, 5'-AGGTTATTGAGGAGTCCCGCGCAGAG-3'.

miRs from tissues and serums were prepared using a miRNeasy Mini Kit (Qiagen, Valencia, CA, USA). The expression of mature miRNA was detected using TaqMan MicroRNA Assays that were specific for miR-187 (hsa-miR-187) (Applied Biosystems, Foster City, CA, USA); each sample was analyzed in triplicate. U6 was used as a control. The primers for miR-187 were: forward, 5'-GACACAGCCTACTTTGGGTC-3' and reverse, 5'-GAAACTGCCTTCCTTGCTACC-3'.

Luciferase activity assay. The 3'-untranslated region (3'-UTR) of human PTRF was amplified by PCR from the A549 cell line. Genomic DNA was templated and cloned in the pMIR-REPORT luciferase vector (Ambion, Carlsbad, CA, USA). The mutant PTRF was created by directed mutagenesis (QuikChange Site-Directed Mutagenesis kit; Agilent Technologies, USA) and constructs were confirmed by sequencing. Twenty-four-well plates were used for culturing the A549 cells. A total of $200 \mathrm{ng}$ of the luciferase reporter constructs and $20 \mathrm{ng}$ of the pRL-CMV Renilla luciferase plasmid were transfected in each well. The Dual-Luciferase Reporter Assay system (Promega, Madison, WI, USA) was used for measuring luciferase activities $48 \mathrm{~h}$ after transfection, according to the manufacturer's instructions.

MTT assay. An MTT assay was used to test the effects of miR-187 on cell proliferation. A549 and H1650 cells were seeded on 96-well plates and miR-187 and PTRF were overexpressed or suppressed. Cells were stained at indicated time points with $100 \mu 1 \mathrm{MTT}$ dye $(0.5 \mathrm{mg} / \mathrm{ml})$ for $4 \mathrm{~h}$ at $37^{\circ} \mathrm{C}$ and then the supernatant was removed and $150 \mu \mathrm{l}$ DMSO (both from Sigma) was added. The absorbance was measured at $570 \mathrm{~nm}$ and the absorbance at $655 \mathrm{~nm}$ was used as the reference wavelength. The MTT assay was performed in triplicate.

Wound healing and invasion assays. Cells were seeded and cultured in 6-well plates. A wound was produced by using a plastic pipette tip when $100 \%$ confluence was reached. The cells were washed with PBS buffer and cultured for $48 \mathrm{~h}$. Cell migration was measured. Wound closure (\%) was calculated as the area of migrated cells divided by the wounded area before the 48 -h culture. A total of $10^{5}$ cells suspended in serum-free media were placed into the upper chamber of an insert with Matrigel and DMEM supplemented with 10\% FBS was inserted into the lower chamber. The cell invasion proceeded for $48 \mathrm{~h}$ into the assay. The cells remaining on the upper surface of the membrane were removed, whereas the cells which had invaded through the membrane were stained with $20 \%$ methanol and $0.2 \%$ crystal violet, imaged, and counted under a microscope.

Protein concentration, western blotting, and antibodies. Protein samples were isolated from the A549 and H1650 cell lines by using RIPA lysis buffer and the protein concentration was measured by BCA assay. The cellular extracts were processed on $10 \%$ SDS-polyacrylamide gel and transferred to a PVDF membrane, which was then probed with antibodies overnight and washed with TBST. And then, it was treated with secondary antibodies and visualized using ECL. GAPDH was used as an internal control and the experiment was performed in triplicate. Antibodies to PTRF and GAPDH were obtained from Cell Signaling Technology. Immunofluorescence staining was performed on NSCLC tissues and adjacent non-tumor tissues.

Bioinformatics and statistical analysis. Target genes of miR-187 were predicted by TargetScan 5.1 (http://www. targetscan.org/), PicTar (http://pictar.mdc-berlin.de/) and miRanda (http://www.microrna.org/). Two-tailed Student's $\mathrm{t}$-test was used to test the differences between the two groups. A Chi-square test was used to evaluate the association between miR-187 and clinicopathological parameters. The Kaplan-Meier method was used for survival analysis and the differences in survival times between groups were evaluated using the log-rank test.

\section{Results}

miR-187 is upregulated in NSCLC tissues and cell lines. Seventy-four paired NSCLC tissues and adjacent non-tumor 
$\mathbf{A}$

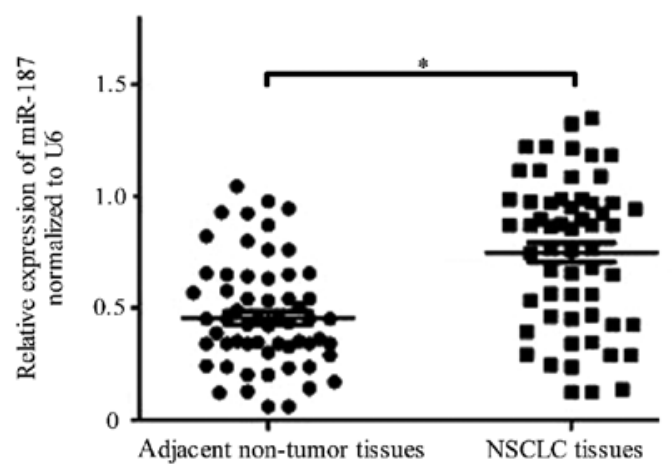

B

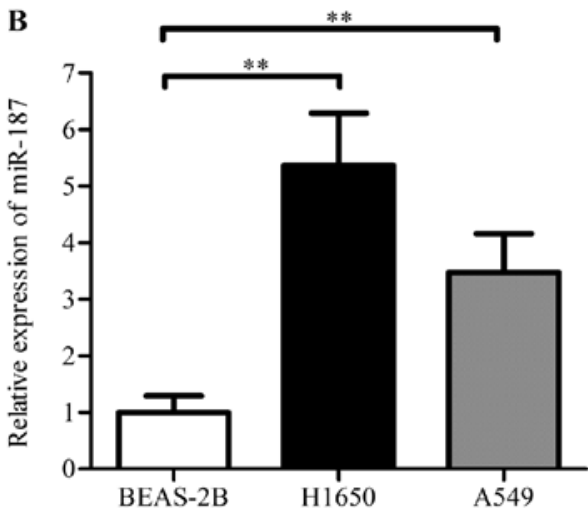

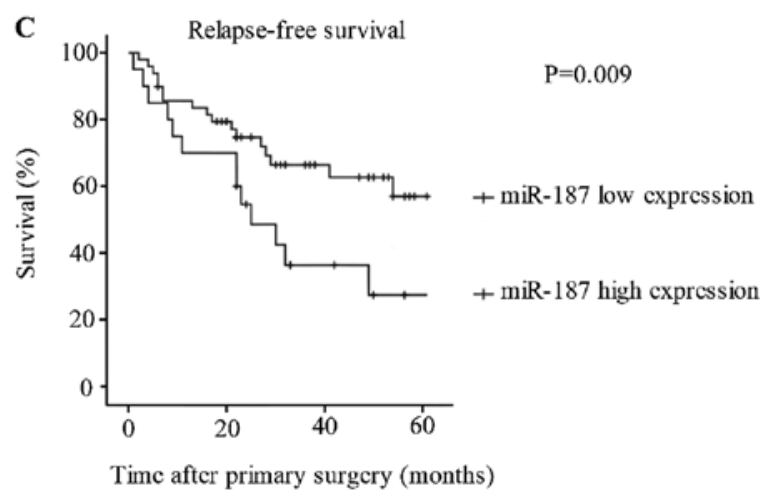

Figure 1. Expression of miR-187 in the NSCLC tissues and cell lines. (A) The expression of miR-187 was measured using RT-PCR in the NSCLC tissues and adjacent normal lung tissues in triplicate. The results were the median values of miR-187 normalized to U6 miRNA. "p<0.05. (B) The expression levels were determined by RT-PCR in triplicate and U6 was used as an internal control. "* $\mathrm{P}<0.01$. (C) The Kaplan-Meier survival curve was drawn in 74 NSCLC tissues grouped by the median levels of miR-187 and the log-rank test was used. miR-187, microRNA-187; NSCLC, non-small cell lung cancer; miRNA, microRNA.

tissue samples were collected from 74 patients and used to measure the miR-187 expression levels using RT-PCR assay. Compared with the adjacent non-tumor lung tissues, the expression level of miR-187 was significantly increased in the NSCLC tissues (Fig. 1A, P<0.05). The expression level of miR-187 in the two cell lines was also measured. The results indicated that both of the cell lines had higher miR-187 expression levels than BEAS-2B, and that $\mathrm{H} 1650$ had an even higher expression level than A549 (Fig. 1B, P<0.05). Collectively, the data suggested that upregulation of miR-187 occurred in the process of NSCLC.

Seventy-four patients were divided into two groups based on the median (0.71) miR-187 level. These groups consisted of a low-miR-187 group (relative expression level $<0.71,30$ cases) and a high-miR-187 group (relative expression level $>0.71$, 44 cases). Clinicopathological parameters were analyzed as shown in Table I. Significant associations between miR-187 expression and metastasis (TNM) stage and lymph node metastasis were observed $(\mathrm{P}<0.05)$. However, associations between miR-187 expression and age and gender were not observed (both $\mathrm{P}>0.05$ ). These data indicated that miR-187 may be involved in NSCLC metastasis and progression.

All patients were followed up to determine the survival time. Kaplan-Meier survival analysis was used to analyze the association between survival time and the expression level of miR-187. The results showed that the patients with a high expression of miR-187 were associated with a shorter overall survival time (Fig. 1C; log-rank test, $\mathrm{P}<0.01$ ). This result
Table I. Association of miR-187 expression with the clinicopathological factors of 74 NSCLC patients.

\begin{tabular}{lcccc}
\hline & \multicolumn{3}{c}{ miR-187 subgroups } & \\
\cline { 3 - 4 } Variables & No. & Low, n (\%) & High, n (\%) & P-value \\
\hline Age (years) & & & & $>0.05$ \\
$<50$ & 13 & $7(53.8)$ & $6(46.2)$ & \\
$\geq 50$ & 61 & $23(37.7)$ & $38(62.3)$ & \\
Gender & & & & $>0.05$ \\
Male & 39 & $14(35.9)$ & $25(64.1)$ & \\
Female & 35 & $16(45.7)$ & $19(54.3)$ & \\
TNM stage & & & & \\
I-II & 58 & $28(48.3)$ & $30(51.7)$ & \\
III-IV & 16 & $2(12.5)$ & $14(87.5)$ & \\
$\begin{array}{l}\text { Lymph node } \\
\text { metastasis }\end{array}$ & & & & $<0.05$ \\
No & 40 & $22(55)$ & $18(45.0)$ & \\
Yes & 34 & $8(23.5)$ & $26(76.5)$ & \\
\hline
\end{tabular}

miR-187, microRNA-187; NSCLC, non-small cell lung cancer.

revealed that patients with higher miR-187 expression level may have a poorer prognosis. 
A

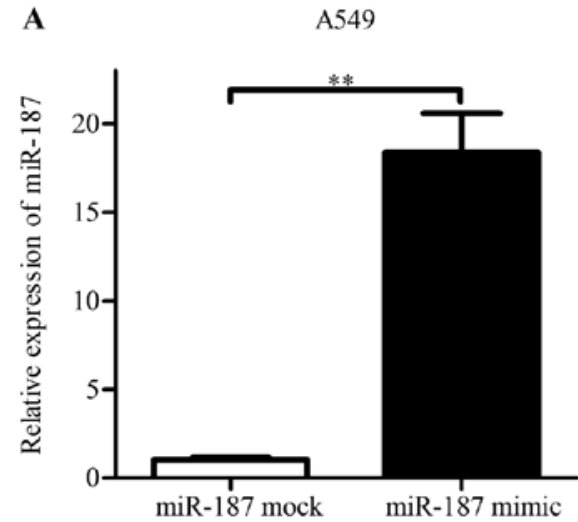

C

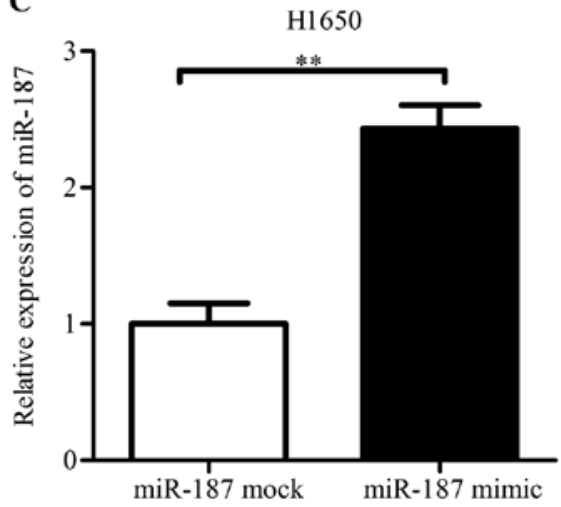

B

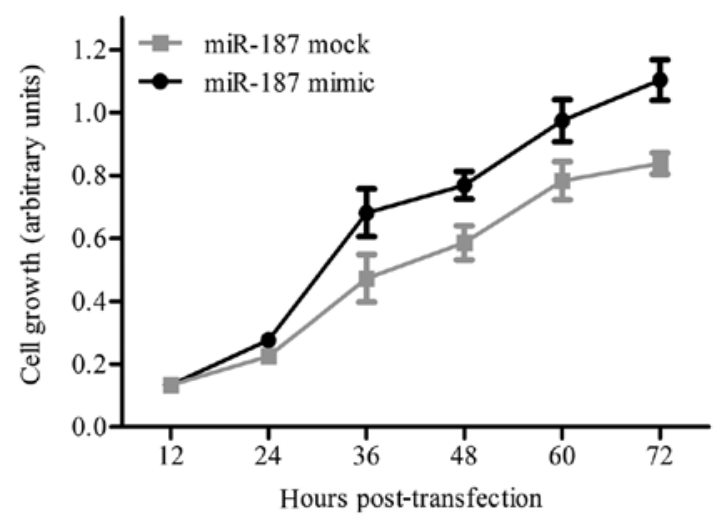

D

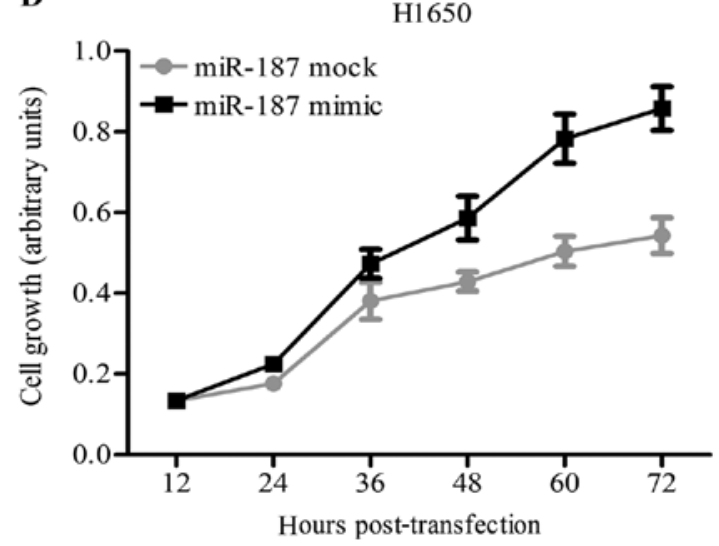

Figure 2. Effect of the overexpression of miR-187 on cell proliferation. (A) The A549 cell line was transfected with a miR-187 mimic and miR-187 expression was assessed using RT-PCR. (B) Cell proliferation was assessed by MTT assay and cell growth was promoted by overexpression of miR-187 in the A549 cells. (C) The H1650 cell line was transfected with a miR-187 mimic and miR-187 expression was assessed using RT-PCR. (D) Cell proliferation was assessed by MTT assay and cell growth was also promoted in the H1650 cells. Each cell type was analyzed in six experiments. ** P<0.01. miR-187, microRNA-187.

miR-187 promotes cell proliferation in the A549 and $H 1650$ cell lines. The A549 and H1650 cell lines were selected for research into the function of miR-187. A miR-187 mimic and miR-187 mock were transfected into the A549 and H1650 cell lines. Cells were collected after $48 \mathrm{~h}$ and the miR-187 levels were measured using RT-PCR. The miR-187 mimic significantly increased the miR-187 expression level in the A549 cell line (Fig. 2A). At first, cell proliferation and growth were measured using the MTT assay to evaluate the function of miR-187. Compared to the control, cell growth of the A549 cells was significantly promoted by the overexpression of miR-187 (Fig. 2B). These results indicated that miR-187 promotes cell growth in the lung cancer cell line. Similar to the results of the A549 cell line, the data obtained from the H1650 cell line showed that overexpression of miR-187 cell lines promote cell growth (Fig. 2C and D). These results indicated that miR-187 performed a positive function in the long-term growth of the NSCLC cells.

miR-187 promotes cell migration and invasion in the H1650 and A549 cell lines. Cell migration and invasion play an important role in cancer metastasis. Some researchers have suggested that miRNAs play important roles in the metastasis of lung cancer cells. Wound healing and Transwell invasion assays were used to test whether miR-187 could impact the migration and invasion of the NSCLC cell lines.
At first, the wound closure rates of the A549 and H1650 cells were measured after a 48-h transfection with the miR-187 mimics. As shown in Fig. 3A, the closure rate was increased in the A549 cell line after transfection with the miR-187 mimics. The rate for the cells transfected with the control mimics only increased to 0.31-fold, and the rate for the cells transfected with the miR-187 mimics was increased to 0.67-fold (Fig. 3B). Similarly, this phenomenon was also observed in the H1650 cell line (Fig. 3C). These results indicated that miR-187 promotes cell migration in the $\mathrm{H} 1650$ and A549 cell lines.

Cell invasion was measured by Transwell invasion assays. As shown in Fig. 4A, the number of invasive cells were increased in the A549 cell line after transfection with the miR-187 mimics. Compared to the control mimics, the invasive ability was increased to 0.92 -fold after transfection with the miR-187 mimics in the A549 cell line (Fig. 4B). Similarly, this phenomenon also was observed in the H1650 cell line (Fig. 4C). These results indicated that miR-187 functioned as a tumor promoter that promoted the growth, migration and invasion of the A549 and H1650 cells.

PTRF is identified as a direct target of miR-187 in the NSCLC cell line. TargetScan, PicTar, and miRanda were used to find the potential target and PTRF was regarded as the candidate gene. PTRF is also known as cavin-1 and it has been reported that its expression is downregulated in 
A

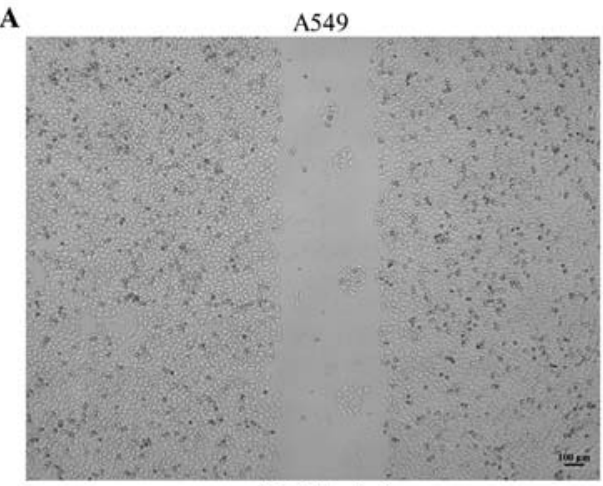

miR-187 mimic

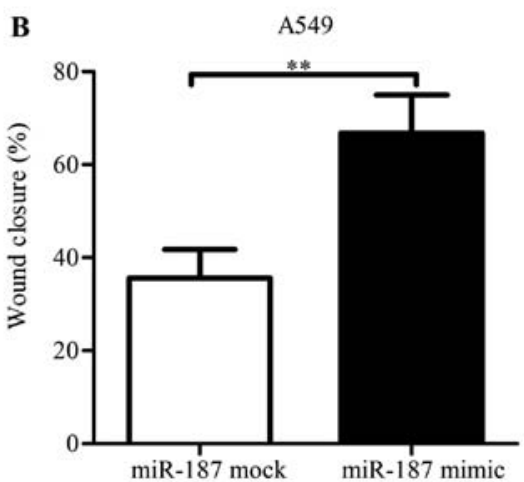

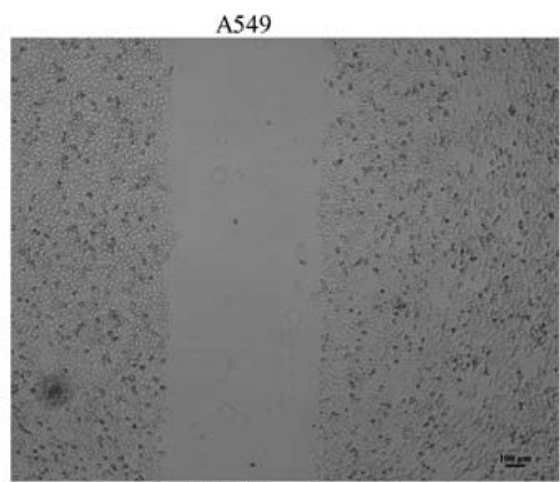

miR-187 mock

C

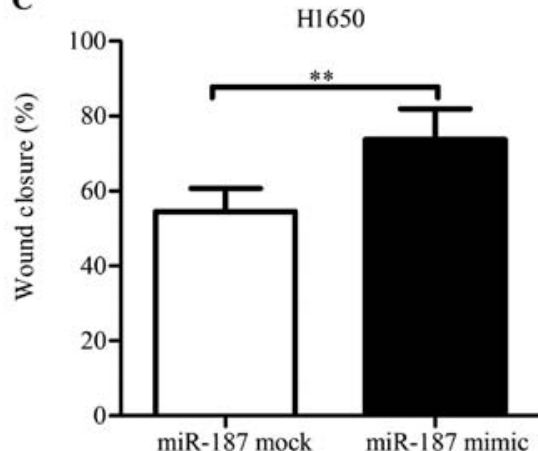

Figure 3. Effect of miR-187 on cell migration in the H1650 and A549 cell lines. (A) The A549 cell line was transfected with miR-187 or control mimics and the migration was investigated using wound healing assays. (B) The A549 cell line was transfected with miR-187 or control mimics in 6-well plates and the wound closure rate was measured at six different positions. (C) The H1650 cell line was transfected with miR-187 or control mimics in 6-well plates and the wound closure rate was measured at six different positions. The wound closure rate was measured by ImageJ after a $48-\mathrm{h}$ transfection. ${ }^{* * *} \mathrm{P}<0.01$. miR-187, microRNA-187.

A

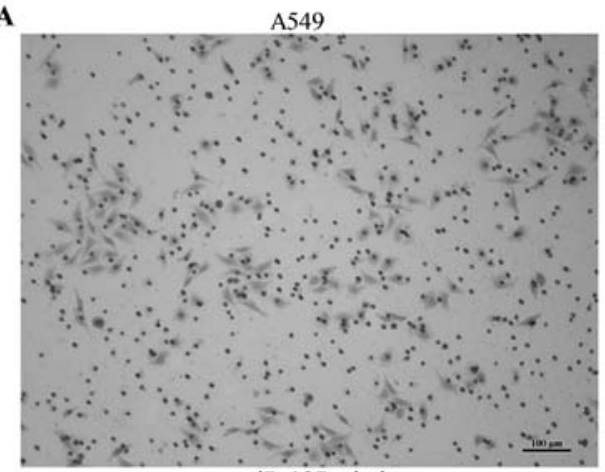

miR-187 mimic

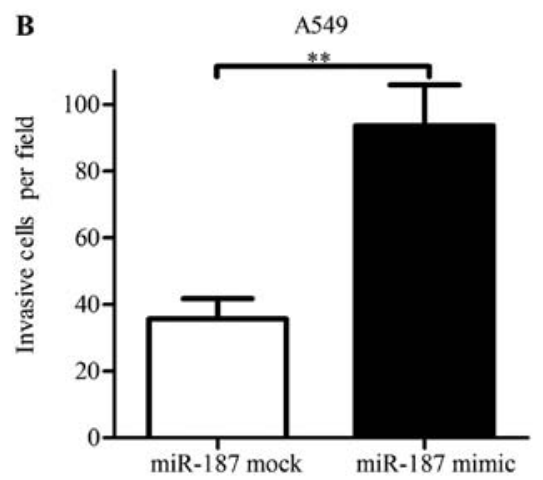

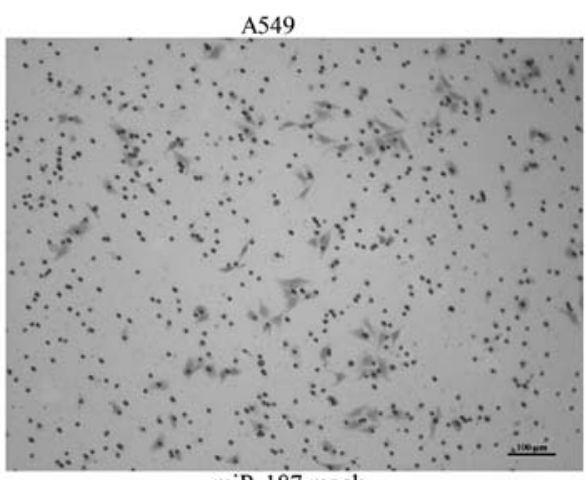

miR-187 mock

C

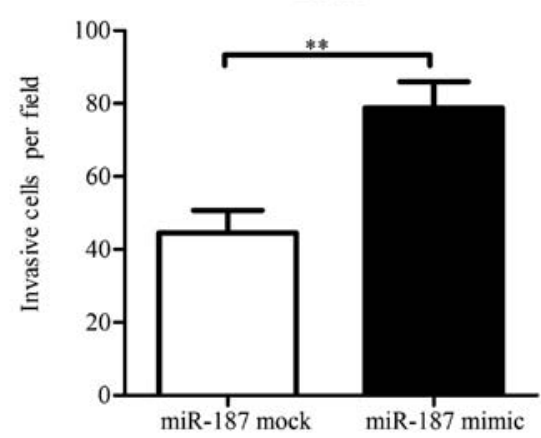

Figure 4. Effect of miR-187 on cell invasion in the H1650 and A549 cell lines. (A) The A549 cells were transfected with miR-187 or control mimics and $10^{5}$ cells were suspended in serum-free media and placed in the upper chamber of an insert precoated with Matrigel. The cells that invaded through the membrane were stained with crystal violet. (B) The A549 cells were transfected with miR-187 or control mimics and the invaded cells were counted at six different positions after $48 \mathrm{~h}$. (C) The H1650 cells were also transfected with miR-187 or control mimics and the invasion assay was performed. The invaded cells were counted under a microscope. ${ }^{* *} \mathrm{P}<0.01$. miR-187, microRNA-187. 
A
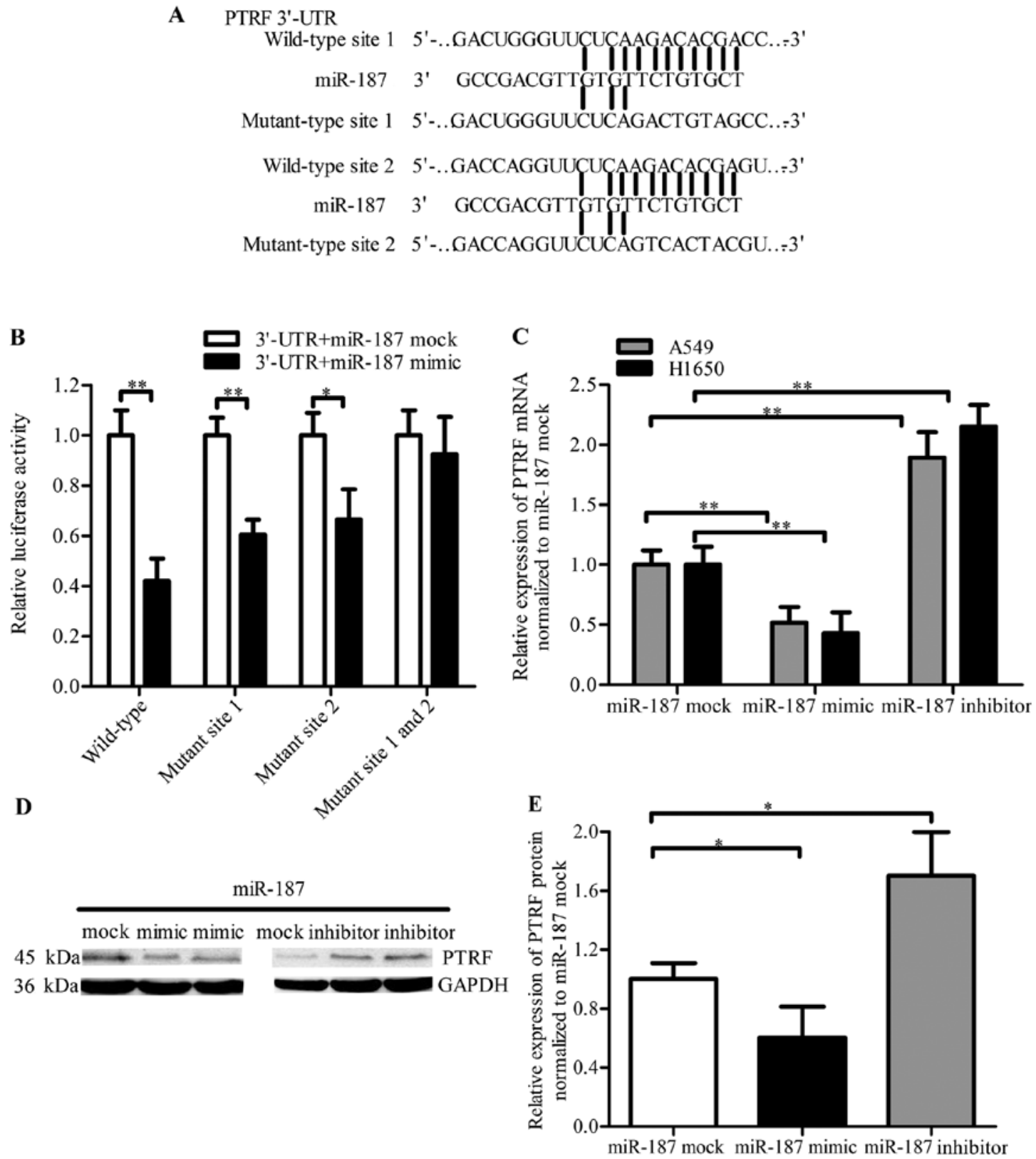

Figure 5. The PTRF gene is directly targeted by miR-187. (A) The 3'-UTR of PTRF and its two binding site fragments and its mutation. Two predicting binding sites were mutant by directed mutagenesis and constructs were confirmed by sequencing. (B) The relative activities were determined using the Dual-Luciferase Reporter Assay System. They were generated from the luciferase reporter plasmids containing wild-type or mutant 3'-UTR of PTRF in the A549 cells with the miR-187 or control mimics. Luciferase activity of the reporter protein was assessed. "P<0.05. (C) RT-PCR analysis was used to detect the mRNA level of PTRF. GAPDH was used as control and the expression levels were normalized to GAPDH. (D) Western blotting was used to analyze the protein level of PTRF. GAPDH was used as a control and the expression levels were normalized to GAPDH. (E) The expression levels of PTRF were decreased following transfection with the miR-187 mimics and increased significantly when transfected with the miR-187 inhibitor. The protein level of PTRF was recorded as a grayscale value and analyzed using ImageJ software. "P $<0.05$. PTRF, polymerase I and transcript release factor; miR-187, microRNA-187; 3'-UTR, 3'-untranslated region; mRNA, messenger RNA; GAPDH, glyceraldehyde-3-phosphate dehydrogenase.

lung cancer samples. Moreover, the overexpression of PTRF reduces the cell migration and knockdown of PTRF leads to increased migration. All evidence suggested that PTRF plays an important role in lung cancer cell migration and it was selected for further research.

There are two binding sites among the wild-type of 3'-UTR. Site 1 and 2 were potential target sites. 3'-UTR of PTRF and three mutant types were fused directly downstream of the firefly luciferase gene, respectively. The complementary sequences are described in Fig. 5A. Wild-type and its mutant types of 3'-UTR of PTRF were constructed in a pMIR-REPORT vector. pMIR-WT, pMIR-mutant 1, pMIR-mutant 2 and pMIR-mutant 1 and 2 were constructed and co-transfected with the miR-187 mimics and control into the A549 cells. Compared with the control, the luciferase activity of the reporter protein was decreased to 0.41 -fold following co-transfection with the miR-187 mimics and pMIR-WT in the A549 cells. Mutation of binding site 1 or 2 increased the luciferase activity of the reporter protein encoded by the gene in the vector to 0.60 - and 0.66 -fold, respectively. Compared with only one 
A

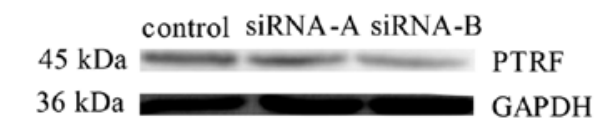

C

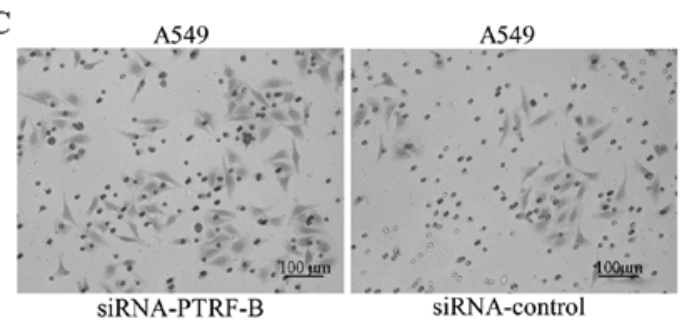

$\mathbf{E}$

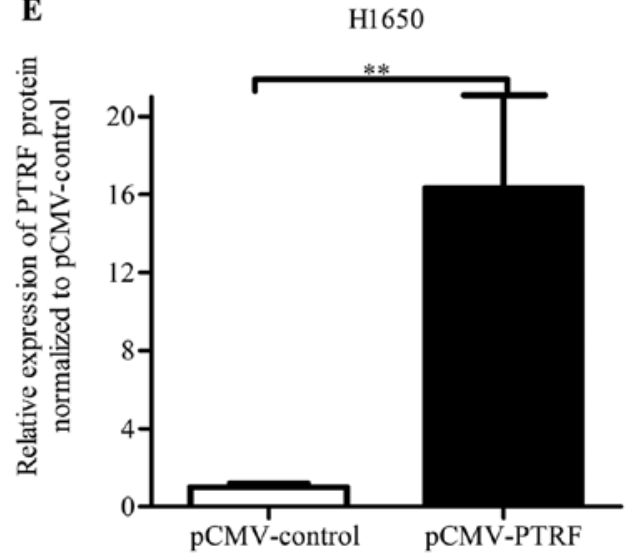

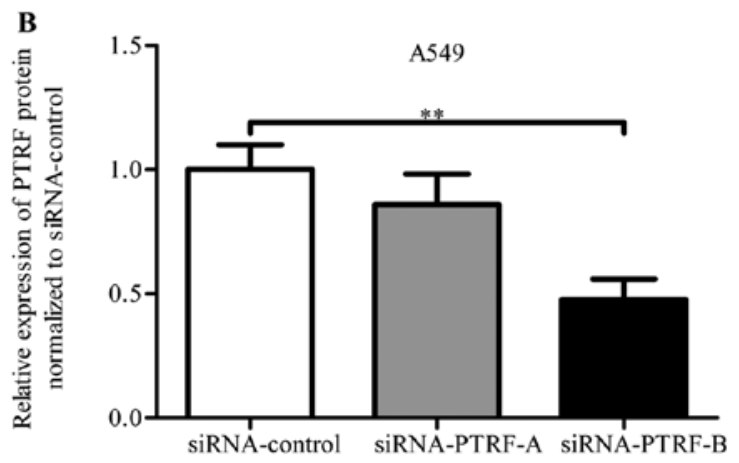

D

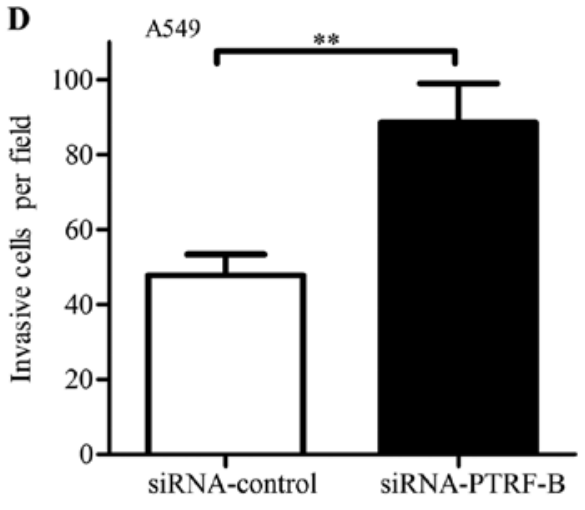

$\mathbf{F}$

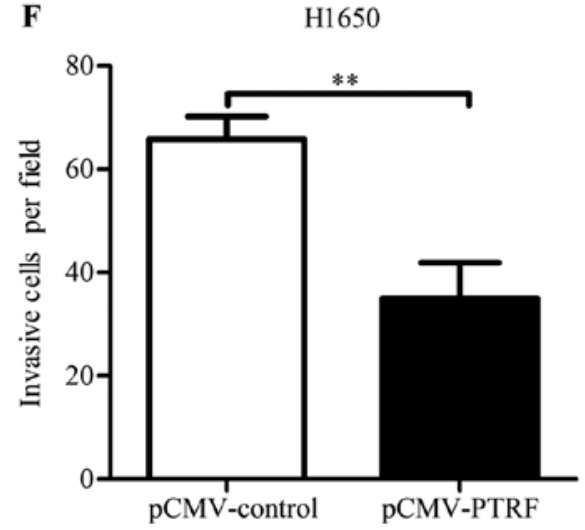

Figure 6. PTRF suppresses lung cancer cell invasion in the A549 and H1650 cell lines. (A) siRNAs and the control were transfected in the A549 cells. Two days post-transfection, the cellular extracts were examined using a PTRF antibody and only one siRNA was selected for further research. (B) Western blotting was used to analyze the protein level of PTRF. The relative expression level of PTRF was decreased in cells following transfection with siRNA-PTRF. The protein level of PTRF was recorded as a grayscale value and analyzed using ImageJ software. (C) The A549 cell line was transfected with siRNA-PTRF or siRNA-control. A total of $10^{5}$ cells were suspended in a serum-free media and placed in the upper chamber of an insert precoated with Matrigel. The cells that invaded through the membrane were stained with crystal violet. (D) The invaded cells were counted at six different positions after $48 \mathrm{~h}$. (E) The H1650 cells were transfected with pCMV-control and pCMV-PTRF. Two days post-transfection, the cellular extracts were examined using a PTRF antibody. The relative expression level of PTRF was increased in cells following transfection with pCMV-PTRF. (F) The invaded cells were counted at six different positions after $48 \mathrm{~h}$ in the H1650 cells. ${ }^{* *} \mathrm{P}<0.01$. PTRF, polymerase I and transcript release factor; siRNA, small interfering RNA.

site mutation, both site 1 and site 2 mutations increased the luciferase activity of reporter protein to 0.92-fold (Fig. 5B). These results indicated that miR-187 inhibited the luciferase activity of the reporter protein by directly binding to the two binding sites and the binding may be affected by mutations. The two binding sites had synergism or summation action. RT-PCR was used to test the PTRF mRNA relative level (Fig. 5C). The results of PTRF mRNA level revealed that transfection with the miR-187 mimics decreased the expression level of PTRF and the miR-187 inhibitor increased the expression level of PTRF to 2.4-fold in the H1650 cells and 1.8-fold in the A549 cells. The protein level of PTRF was assessed using western blotting (Fig. 5D and E). The results revealed that relative level of PTRF normalized to GAPDH decreased to 0.60 -fold in the A549 cells transfected with the miR-187 mimics (Fig. 5E) and the expression levels increased to 1.7 -fold with the miR-187 inhibitor. Notably, the increased level of miR-187 in the lung cancer cells was confirmed. It may have an impact on endogenous PTRF expression and may significantly silence its mRNA and protein.

PTRF suppresses lung cancer cell invasion in vitro. siRNA-PTRF was used to knock down the expression of PTRF in the A549 cell line. The H1650 cells had a higher miR-187 expression level than the A549 cells and the expression levels of the PTRF protein were significantly downregulated 


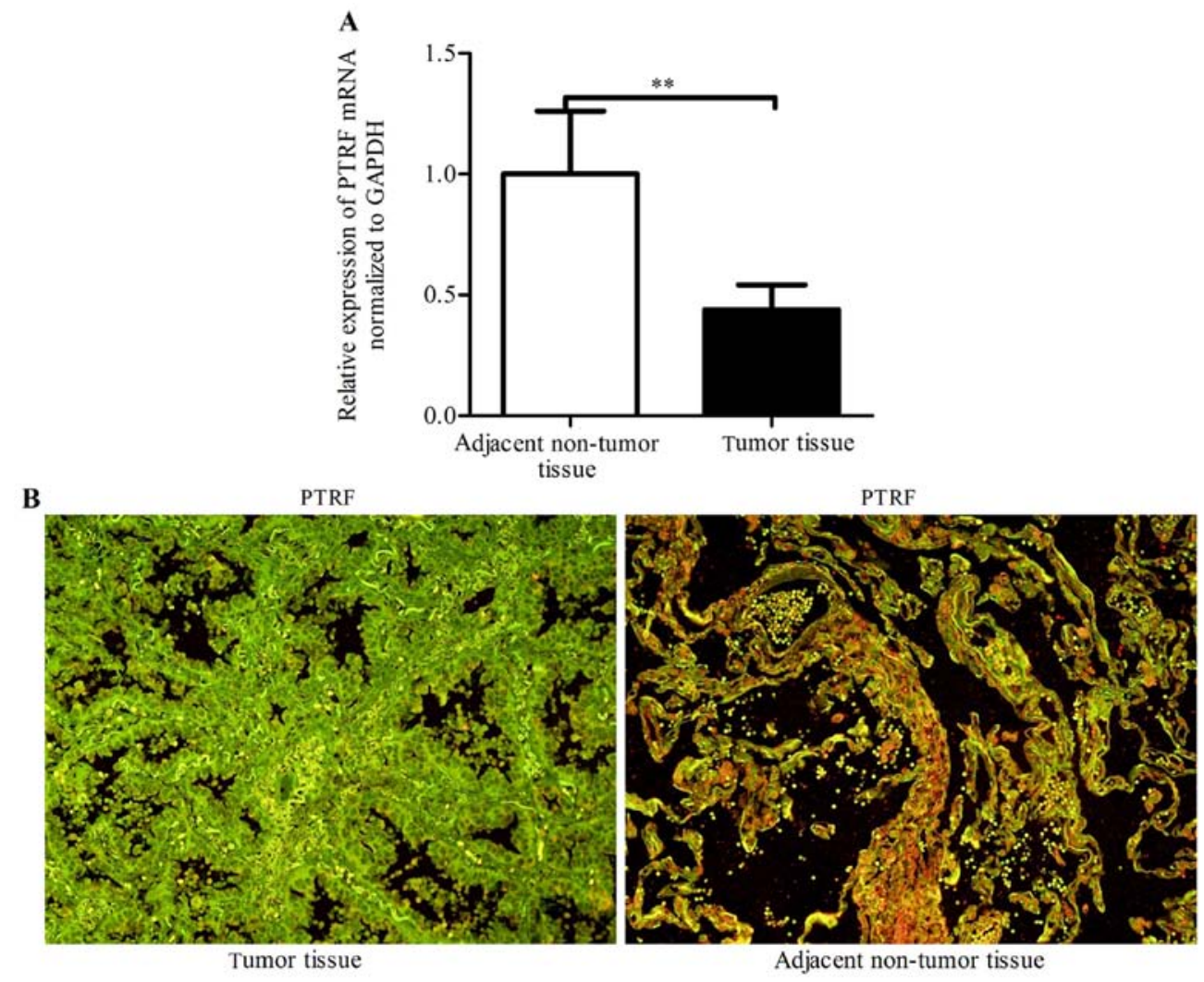

Figure 7. Analysis of PTRF in NSCLC tissues and adjacent non-tumor tissues. (A) Determination of the relative mRNA level of PTRF in 74 pairs of lung cancer tissues and their matched normal tissues using RT-PCR. GAPDH mRNA was used as a control. (B) The qualitative analysis of the PTRF protein in the NSCLC tissues and their adjacent non-tumor tissues was performed using immunofluorescence staining. The color orange indicates the expression of PTRF. ${ }^{* *} \mathrm{P}<0.01$. PTRF, polymerase I and transcript release factor; NSCLC, non-small cell lung cancer; mRNA, messenger RNA; GAPDH, glyceraldehyde-3-phosphate dehydrogenase.

following transfection with siRNA-PTRF (Fig. 6A and B). Moreover, Transwell assay was used to detect the cell invasion. The results revealed that cell invasion in cells transfected with the siRNA-PTRF was higher than that noted in the control siRNA cells (Fig. 6C and D). The results demonstrated that PTRF was a suppressor in lung cancer cells and knockdown of PTRF promoted lung cancer cell invasion in the A549 cells.

To detect the effect of the overexpression of PTRF on NSCLC, the H1650 cell line was selected for further research. The H1650 cells were transfected with a pCMV-PTRF vector. Western blotting revealed that the expression of PTRF was significantly increased (Fig. 6E). Cell invasion was detected (Fig. 6F) and the number of invading cells were decreased, compared with the control pCMV vector. These results indicated that overexpression of PTRF had a negative effect on lung cancer cell invasion.

PTRF is downregulated in human NSCLC tissues. As the expression level of miR-187 was upregulated in the NSCLC tissues and cell lines, PTRF could be downregulated in the human NSCLC tissues. To test this hypothesis, lung cancer tissues and adjacent normal lung tissues were collected to assess the expression. RT-PCR was used to analyze the PTRF expression levels and the results revealed that the PTRF mRNA level in the cancer tissues was significantly lower than that in their adjacent normal lung tissues (Fig. 7A). Immunohistochemical staining was used to detect the expression in cancer tissues.
As shown in Fig. 7B, the expression of the PTRF protein in lung cancer tissues was significantly weaker than that in the adjacent normal lung tissues. All of these results revealed that the expression of PTRF was downregulated in the lung cancer tissues.

\section{Discussion}

Lung cancer development is a multistep process and miRNAs play an important role in cancer cell proliferation, metastasis and invasion (2-8). In this study, the expression of miR-187 was upregulated in NSCLC tumor tissues and cell lines. Moreover, overexpression of miR-187 promoted lung cancer cell proliferation, metastasis, and invasion. A target of miR-187 was predicted and PTRF was identified as a new direct target of miR-187. PTRF knockdown using siRNAs promoted cell invasion. Additionally, PTRF expression was decreased in the NSCLC cancer tissues. These results indicated that miR-187 may be a tumor promoter in NSCLC via the targeting of PTRF. This is the first study to show that loss of expression of PTRF in NSCLC tumor tissues was mediated by miR-187.

Previous studies have demonstrated that miR-187 impacts cell invasion, proliferation, and migration in cancers (14-18). However, the reported results are conflicting. Notable, Zhao et al (18) demonstrated that miR-187 was downregulated and negatively regulated B7 homolog 3 (B7-H3) expression in clear cell renal cell carcinoma cells. Chao et al (15) also 
demonstrated that miR-187 was upregulated and negatively regulated disabled homolog-2 (Dab2) expression in ovarian cancer cells. The evidence indicates that the expression of miR-187 is heterogeneous and that research on a larger sample size is needed. Considering that the study reported by Mirzadeh Azad et al had a small sample size (16), 61 patients with adenocarinoma, 11 patients with squamous cell carcinoma, and 2 patients with large cell carcinoma were included in this study. Different expression levels of miR-187 were observed. It was also revealed that the role of miR-187 in NSCLC remains uncertain and needs further research.

Compared to other research, a new target gene was observed in this study. A 3'-UTR sequence of PTRF was predicted as a target of miR-187 using prediction software. Luciferase reporter assay was performed and the results showed that miR-187 directly targeted the 3'-UTR of PTRF.PTRF (cavin-1) is essential for RNA transcription (19) and formation and function of caveolae (20). These invaginations of the cell surface are associated with processes of vesicular transport, cholesterol homeostasis, and signal transduction (21). The absence of expression of PTRF in prostate cancer was found to be related with progression of this disease (22), and it was demonstrated that its expression decreased the migration of prostate cancer cells $(23,24)$. Bai et al reported that PTRF was downregulated in breast cancer cell lines and breast tumor tissues (25). PTRF has been described in cells that overexpress EGFR, which suggests a function in this signaling pathway (26). Our results indicated that PTRF expression was decreased in the NSCLC samples.

On the one hand, the loss of expression of PTRF may affect the activity of caveolin-1, and non-caveolar caveolin-1 may exert its tumor-promoting effects. It may also affect the interaction with PTRF-EGFR and the EGFR pathway may be deregulated in the NSCLC samples. This suggests a role of PTRF in NSCLC development.

On the other hand, PTRF expression may enable caveolae formation and may trap caveolin-1 in caveolae, which may affect the cytokines and chemokines (27). Absence of the expression of PTRF was found to lead to overexpression of various cytokines, which may exert a tumor-promoting effects in the cells. Lack of expression of PTRF may affect cancer cholesterol transport (28), which may lead to lung cancer aggressiveness and invasion (23,29-31). PTRF may also modulate the expression of genes that have key roles in lung cancer invasion, such as MMP-9 (30).

There are some limitations in this study. First, in vivo angiogenesis and lymphangiogenesis models were not performed. Second, the sample size was not large and this topic needs further research. Third, there are many target genes of miR-187 and we were not able to verify all of them and there may be other target genes involved in lung cancer. In our study, we provide convincing evidence that miR-187 functions as a cancer promoter in NSCLC, by targeting PTRF expression.

\section{References}

1. Begum S, Hayashi M, Ogawa T, Jabboure FJ, Brait M, Izumchenko E, Tabak S, Ahrendt SA, Westra WH, Koch W, et al: An integrated genome-wide approach to discover deregulated microRNAs in non-small cell lung cancer: Clinical significance of $m i R-23 b-3 p$ deregulation. Sci Rep 5: 13236, 2015.
2. Zhang ZY, Fu SL, Xu SQ, Zhou X, Liu XS, Xu YJ, Zhao JP and Wei S: By downregulating Ku80, hsa-miR-526b suppresses non-small cell lung cancer. Oncotarget 6: 1462-1477, 2015.

3. Chen C, Zhao Z, Liu Y and Mu D: MicroRNA-99a is downregulated and promotes proliferation, migration and invasion in non-small cell lung cancer A549 and H1299 cells. Oncol Lett 9: 1128-1134, 2015.

4. Zhang B, Liu T, Wu T, Wang Z, Rao Z and Gao J: microRNA-137 functions as a tumor suppressor in human non-small cell lung cancer by targeting SLC22A18. Int J Biol Macromol 74: 111-118, 2015.

5. Ai C, Jiang R, Fu L and Chen Y: MicroRNA-495 mimics delivery inhibits lung tumor progression. Tumour Biol 36: 729-735, 2015.

6. Shi WY, Liu KD, Xu SG, Zhang JT, Yu LL, Xu KQ and Zhang TF: Gene expression analysis of lung cancer. Eur Rev Med Pharmacol Sci 18: 217-228, 2014.

7. Ohdaira H, Sekiguchi M, Miyata $K$ and Yoshida K: MicroRNA-494 suppresses cell proliferation and induces senescence in A549 lung cancer cells. Cell Prolif 45: 32-38, 2012.

8. Liang J, Zhang Y, Jiang G, Liu Z, Xiang W, Chen X, Chen Z and Zhao J: MiR-138 induces renal carcinoma cell senescence by targeting EZH2 and is downregulated in human clear cell renal cell carcinoma. Oncol Res 21: 83-91, 2013.

9. Li Z, Lei H, Luo M, Wang Y, Dong L, Ma Y, Liu C, Song W, Wang F, Zhang J, et al: DNA methylation downregulated mir-10b acts as a tumor suppressor in gastric cancer. Gastric Cancer 18: 43-54, 2015.

10. Zhang J, Xu L, Yang Z, Lu H, Hu D, Li W, Zhang Z, Liu B and Ma S: MicroRNA-10b indicates a poor prognosis of non-small cell lung cancer and targets E-cadherin. Clin Transl Oncol 17: 209-214, 2015.

11. Zhao G, Liu L, Zhao T, Jin S, Jiang S, Cao S, Han J, Xin Y, Dong Q, Liu X, et al: Upregulation of miR-24 promotes cell proliferation by targeting NAIF1 in non-small cell lung cancer. Tumour Biol 36: 3693-3701, 2015.

12. Xie X, Liu H, Wang M, Ding F, Xiao H, Hu F, Hu R and Mei J: miR-342-3p targets RAP2B to suppress proliferation and invasion of non-small cell lung cancer cells. Tumour Biol 36: 5031-5038, 2015.

13. Zhang W, Liu K, Liu S, Ji B, Wang Y and Liu Y: MicroRNA-133a functions as a tumor suppressor by targeting IGF-1R in hepatocellular carcinoma. Tumour Biol 36: 9779-9788, 2015.

14. Mulrane L, Madden SF, Brennan DJ, Gremel G, McGee SF, McNally S, Martin F, Crown JP, Jirström K, Higgins DG, et al: miR-187 is an independent prognostic factor in breast cancer and confers increased invasive potential in vitro. Clin Cancer Res 18: 6702-6713, 2012.

15. Chao A, Lin CY, Lee YS, Tsai CL, Wei PC, Hsueh S, Wu TI, Tsai CN, Wang CJ, Chao AS, et al: Regulation of ovarian cancer progression by microRNA-187 through targeting Disabled homolog-2. Oncogene 31: 764-775, 2012.

16. Mirzadeh Azad F, Naeli P, Malakootian M, Baradaran A, Tavallaei M, Ghanei M and Mowla SJ: Two lung development-related microRNAs, miR-134 and miR-187, are differentially expressed in lung tumors. Gene 577: 221-226, 2016.

17. Yan ZX, Wu LL, Xue K, Zhang QL, Guo Y, Romero M, Leboeuf C, Janin A, Chen SJ, Wang L, et al: MicroRNA187 overexpression is related to tumor progression and determines sensitivity to bortezomib in peripheral T-cell lymphoma. Leukemia 28: 880-887, 2014.

18. Zhao J, Lei T, Xu C, Li H, Ma W, Yang Y, Fan S and Liu Y: MicroRNA-187, down-regulated in clear cell renal cell carcinoma and associated with lower survival, inhibits cell growth and migration though targeting B7-H3. Biochem Biophys Res Commun 438: 439-444, 2013.

19. Jansa P and Grummt I: Mechanism of transcription termination: PTRF interacts with the largest subunit of RNA polymerase I and dissociates paused transcription complexes from yeast and mouse. Mol Gen Genet 262: 508-514, 1999.

20. Hill MM, Bastiani M, Luetterforst R, Kirkham M, Kirkham A, Nixon SJ, Walser P, Abankwa D, Oorschot VM, Martin S, et al: PTRF-Cavin, a conserved cytoplasmic protein required for caveola formation and function. Cell 132: 113-124, 2008.

21. Cohen AW, Hnasko R, Schubert W and Lisanti MP: Role of caveolae and caveolins in health and disease. Physiol Rev 84: 1341-1379, 2004.

22. Gould ML, Williams G and Nicholson HD: Changes in caveolae, caveolin, and polymerase 1 and transcript release factor (PTRF) expression in prostate cancer progression. Prostate 70: 1609-1621, 2010. 
23. Aung CS, Hill MM, Bastiani M, Parton RG and Parat MO PTRF-cavin-1 expression decreases the migration of PC3 prostate cancer cells: Role of matrix metalloprotease 9. Eur J Cell Biol 90: 136-142, 2011.

24. Shen J, Behrens C, Wistuba II, Feng L, Lee JJ, Hong WK and Lotan R: Identification and validation of differences in protein levels in normal, premalignant, and malignant lung cells and tissues using high-throughput Western Array and immunohistochemistry. Cancer Res 66: 11194-11206, 2006.

25. Bai L, Deng X, Li Q, Wang M, An W, Deli A, Gao Z, Xie Y, Dai Y and Cong YS: Down-regulation of the cavin family proteins in breast cancer. J Cell Biochem 113: 322-328, 2012.

26. Moon H, Lee CS, Inder KL, Sharma S, Choi E, Black DM, Lê Cao KA, Winterford C, Coward JI, Ling MT, et al; Australian Prostate Cancer BioResource: PTRF/cavin-1 neutralizes non-caveolar caveolin-1 microdomains in prostate cancer. Oncogene 33: 3561-3570, 2014.

27. Guha U, Chaerkady R, Marimuthu A, Patterson AS, Kashyap MK, Harsha HC, Sato M, Bader JS, Lash AE, Minna JD, et al: Comparisons of tyrosine phosphorylated proteins in cells expressing lung cancer-specific alleles of EGFR and KRAS. Proc Natl Acad Sci USA 105: 14112-14117, 2008.
28. Rossato M, Curtale G, Tamassia N, Castellucci M, Mori L, Gasperini S, Mariotti B, De Luca M, Mirolo M, Cassatella MA, et al: IL-10-induced microRNA-187 negatively regulates TNF- $\alpha$, IL- 6 , and IL-12p40 production in TLR4-stimulated monocytes. Proc Natl Acad Sci USA 109: E3101-E3110, 2012.

29. Fu Y, Hoang A, Escher G, Parton RG, Krozowski Z and Sviridov D: Expression of caveolin-1 enhances cholesterol efflux in hepatic cells. J Biol Chem 279: 14140-14146, 2004.

30. Lee BH, Taylor MG, Robinet P, Smith JD, Schweitzer J, Sehayek E, Falzarano SM, Magi-Galluzzi C, Klein EA and Ting AH: Dysregulation of cholesterol homeostasis in human prostate cancer through loss of ABCA1. Cancer Res 73: 1211-1218, 2013.

31. Nassar ZD, Hill MM, Parton RG and Parat MO: Caveola-forming proteins caveolin-1 and PTRF in prostate cancer. Nat Rev Urol 10: 529-536, 2013. 